\title{
Real Time Monitoring of Posture to Improve Ergonomics
}

\author{
${ }^{1}$ Manju Gopinath and ${ }^{2}$ Angeline Kirubha \\ Department of Biomedical Engineering, SRM University, India \\ ${ }^{1}$ manju_gopinath@srmuniv.edu.in; ${ }^{2}$ angeline.sp@ktr.srmuniv.ac.in
}

\begin{abstract}
Back pain is one of humanities most frequent complaints. While dangerous diseases are being combatted on a daily basis, something as simple as bad posture is often overlooked. Bad posture can start off as a simple habit and soon lead to chronic back pain. Thus it is essential to maintain an ideal posture so as to reduce stress on the body. In this paper, monitoring of posture is done using flex sensor placed along the spine to detect the movement or bend. As posture changes from 'ideal' to 'poor', the user is alerted through a buzzer and LCD. A microcontroller is used to set the limits of ideal posture, which can be user specific. A load cell is used to monitor the variation in body weight and spine stress for poor and ideal postures under sitting and standing cases. The proposed system aims to implement a simple posture monitoring and correction system that can be set up at the work place with ease. This can serve to prevent cases of scoliosis and other spine issues which often start with poor posture.
\end{abstract}

Keywords: posture; flex sensor; load cell; ergonomics.

\section{Introduction}

Back pain is one of humanities most frequent complaints. In industrialized countries $80 \%$ of the population will experience back pain at some point in their life. The number of people with back pain increases with advancing age, starting in school children and peaking in adults of 35 to 55 years of age [1].

Ideal posture indicates proper alignment of the body's segments such that least amount of energy is required to maintain a desired position. Good posture optimizes breathing and improves the circulation of bodily fluids [2].

After a time, poor posture feels normal and continues to regress further from the correct posture. When poor posture feels normal to a person, it becomes harder to correct because the muscle memory now stores the information needed for poor posture and disposes of the memory for correct posture [3]. Poor posture will continue to digress the longer it is left uncorrected. With a simple posture monitoring and correcting system as described in this paper, one can monitor the posture easily and thereby correct it on the long run.

\section{The Existing Systems}

The existing systems for posture monitoring are complicated systems with markers and IR cameras set up and incorporates a gait lab for posture evaluation. Such a system is difficult to set up for continuous 
Manju Gopinath and Angeline Kirubha; Real Time Monitoring of Posture to Improve Ergonomics. Journal of Biomedical Engineering and Medical Imaging, Volume 2, No 2, April (2015) , pp 22-25

monitoring of posture. Other systems use accelerometers and gyroscopes placed on the patients back and shoulders to detect the spine movement. However, the data obtained tends to be too sensitive to properly monitor the postures [4]. Smart vests have been developed for posture monitoring where inductive sensors were sown into a t-shirt. But drawbacks include motion artifacts and wearability of the t-shirt designed [5].

\section{Methodology and Design}

\subsection{Block Diagram of Proposed System}

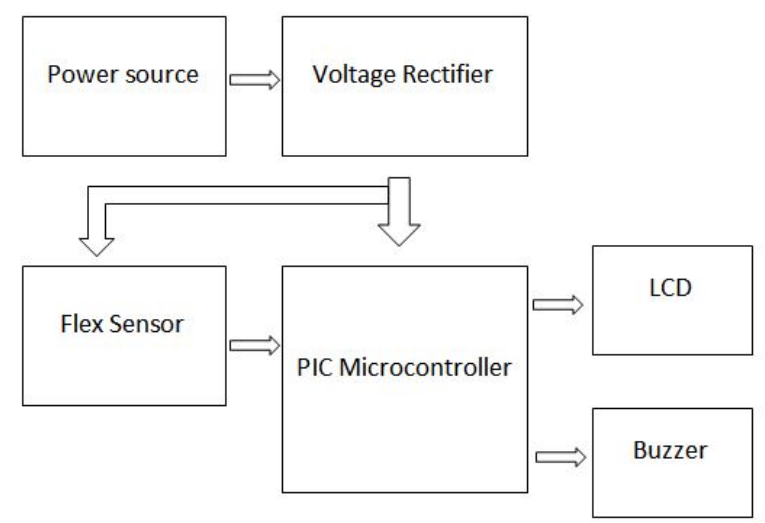

Figure1. Block Diagram of Flex Sensor Module

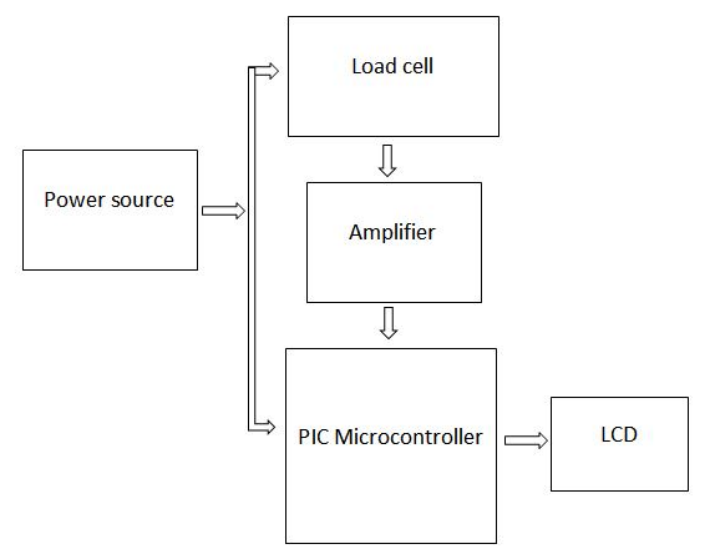

Figure 2. Block Diagram of Load Cell Module

A flex sensor is placed on the subject's back along the curve of the spine in the mid thoracic region. A load cell is placed between platforms on which the subject can stand. Signals from the flex sensor and the load cell are sent to a microcontroller with a built in analog to digital conversion. The microcontroller is programmed to monitor the posture and check for deviation from normal posture and sound the buzzer as an alert. The flex sensor and load cell values are displayed on the LCD. The values are also sent to a computer to store and analyze at a later point of time.

\subsection{Detecting Spine Variation using Flex Sensor}

As the person bends their spine, the flex sensor also bends, causing a change in resistance which is converted to voltage values and digitalized. The microcontroller has a user defined threshold limit which can be determined by trial and error for each subject individually. The microcontroller detects the posture variation and sounds the buzzer when the subjects posture exceeds the threshold limit set by the user. As different people have different body types and spine curvatures, the threshold limit is set depending on the patients comfort level and can be adjusted as required.

\subsection{Detecting the Load Variation using Load Cells}

A load sensor is used to measure the stress that is being exerted on the spine while posture varies from ideal to poor. By this, the subject can determine how much change in load is present on the spine and the whole body. Load cell reading is taken for sitting and standing position and the variation in load while posture is altered can be determined. The values are displayed on an LCD and also sent to a computer to store and analyze at a later point of time. 


\section{Experiment and Analysis}

The flex sensors were positioned on the mid thoracic region for optimal detection and threshold was determined under a relaxed posture while sitting. The posture variation was tested by bending the spine to mimic poor posture condition. The load cell was placed under a platform on which the subject would stand. The variation in load was tested for sitting and standing condition under poor and ideal posture.

Table 1. Detection of Poor Posture under various placements of the sensor.

\begin{tabular}{|l|c|c|}
\hline Sensor Placement & Threshold Range [0 to 50] & Detection of Poor Posture \\
\hline Upper Thoracic & 45 & Buzzer sounds when $>45$ \\
\hline Lower Thoracic & 42 & $42-45$ Unable to detect \\
\hline Mid Back & 47 & Detects from 45 \\
\hline Lumbar & Not definable & Not detectable \\
\hline
\end{tabular}

Sensor placement is best suited for upper thoracic or mid back, depending on the subject. For lower thoracic and lumbar regions, flex sensor is not sensitive enough to detect the bend in spine adequately.

Table 2. Variation of Load under Poor and Normal Posture for Sitting Position.

\begin{tabular}{|l|c|c|c|c|}
\hline Subject & Weight (kg) & $\begin{array}{c}\text { Load in Good } \\
\text { Posture (N) }\end{array}$ & $\begin{array}{c}\text { Load in Poor } \\
\text { Posture (N) }\end{array}$ & $\begin{array}{c}\text { \% Increase in Load } \\
\text { on Spine }\end{array}$ \\
\hline S1 & 52 & 431.5 & 433.2 & 0.39 \\
\hline S2 & 57 & 461 & 464 & 0.65 \\
\hline S3 & 63 & 490 & 492.6 & 0.53 \\
\hline
\end{tabular}

Table 3. Variation of Load under Poor and Normal Posture for Standing Position.

\begin{tabular}{|l|c|c|c|c|}
\hline \multicolumn{1}{|c|}{ Subject } & Weight (kg) & $\begin{array}{c}\text { Load in Good } \\
\text { Posture (N) }\end{array}$ & $\begin{array}{c}\text { Load in Poor } \\
\text { Posture (N) }\end{array}$ & $\begin{array}{c}\text { \% Increase in Load } \\
\text { on Spine }\end{array}$ \\
\hline S1 & 52 & 510 & 519.8 & 1.92 \\
\hline S2 & 57 & 560 & 581 & 3.75 \\
\hline S3 & 63 & 618 & 637.5 & 3.15 \\
\hline
\end{tabular}

During detection of load variation, subjects were made to maintain position without any voluntary movements for proper measurement.

\section{Conclusion}

We were able to implement a simple posture monitoring and correction system using flex sensor and load cell. Flex sensor can be used as a detection of poor posture applicable for cases of scoliosis and other thoracic abnormalities. Load cell readings indicate that $85 \%$ of the body weight is on the buttocks while sitting compared to standing.

In sitting position, poor posture affects the load on the spine by $0.52 \%$ increase in load. In standing position, poor posture has a much more prominent affect, increasing the load on spine by $2.94 \%$. Using the proposed system, posture can be monitored on a continuous basis and load applied on the spine can be minimized.

In the future work, the system can be improved to wirelessly transmit the data to a computer for analysis. The system can also be made more compact so as to monitor posture for additional activities 
Manju Gopinath and Angeline Kirubha; Real Time Monitoring of Posture to Improve Ergonomics. Journal of Biomedical Engineering and Medical Imaging, Volume 2, No 2, April (2015) , pp 22-25

such as walking, running, etc. A custom made flex sensor of greater length will be able to detect variation of entire spine curvature instead of being limited to a small region.

\section{REFERENCES}

[1] Institute for Health Metrics and Evaluation. "2010 Global Burden of Disease Study".

[2] A Patient's Guide to Rehabilitation for Low Back Pain: Understanding the Neutral Spine Position.

[3] Sullivan, Karin Horgan, Jan 1999, 'Perfect Posture', Vegetarian Times 257, p.64.

[4] Bilal El-Sayed, et al., (2011) 'A Novel Mobile Wireless Sensing System for Realtime Monitoring of Posture and Spine Stress', 1st Middle East Conference on Biomedical Engineering (MECBME)

[5] Emilio Sardini, et al., 'Smart Vest for Posture Monitoring in Rehabilitation Exercises', Sensors Applications Symposium (SAS), IEEE. 